\title{
Learning to Forecast the Exchange Rate: Two Competing Approaches.
}

\author{
Paul De Grauwe and Agnieszka Markiewicz*
}

April 2006

\begin{abstract}
In this paper, we investigate the behavior of the exchange rate within the framework of an asset pricing model. We assume boundedly rational agents who use simple rules to forecast the future exchange rate. They test these rules continuously using two learning mechanisms. The first one, the fitness method, assumes that agents evaluate forecasts by computing their past profitability. In the second mechanism, agents learn to improve these rules using statistical methods. First, we find that both learning mechanisms reveal the fundamental value of the exchange rate in the steady state. Second, both mechanisms mimic regularities observed in the foreign exchange markets, namely exchange rate disconnect and excess volatility. Fitness learning rule generates the disconnection at different frequencies, while the statistical method has this ability only at the high frequencies. Statistical learning can produce excess volatility of magnitude closer to reality than fitness learning but can also lead to explosive solutions.
\end{abstract}

\footnotetext{
${ }^{*}$ Center for Economic Studies, Department of Economics, Naamsestraat 69, 3000 Leuven, Belgium, Paul.Degrauwe@econ.kuleuven.ac.be, Agnieszka.Markiewicz@econ.kuleuven.ac.be
} 


\section{Introduction}

Exchange rate economics has been dominated by the rational expectations efficient market theory. As the empirical evidence against this theory has tended to accumulate over time $^{1}$, researchers have increasingly looked for alternative modelling approaches. One of these approaches challenges the assumptions about the way the agents form their expectations. In this paper, we focus on this approach.

We investigate the behavior of the exchange rate within the framework of a standard asset pricing model. We assume that the market expectations, within this model, are formed by boundedly rational agents. We take the view that the rational expectations assumption puts too great an informational burden on individual agents. As argued by Kahneman and Tversky (1973) and Kahneman (2002), agents experience cognitive problems in processing information. As a result, they use simple forecasting rules (heuristics). We assume that they can use two different forecasting rules and combine them to form their expectations about the future exchange rate. The first one will be called a fundamentalist forecasting rule, the second one a chartist rule (technical analysis). Then, we assume that the agents test these rules continuously. This testing procedure is the mechanism by which we introduce discipline on the behavior of individual agents. We specify two alternative testing procedures (learning mechanisms). In the first one, agents select the rules based on a fitness method in the spirit of Brock and Hommes (1997), (1998). This mechanism assumes that agents evaluate forecasts by computing their past profitability. Accordingly, they increase (reduce) the weight of one rule if it is more (less) profitable than the alternative rule. In the second mechanism, agents learn to improve these rules using statistical methods based on the literature of learning in macroeconomics (e.g. Evans and Honkapohja (2001)).

The purpose of this paper is to analyze the behavior of the exchange rate under different learning rules, and to compare the capacity of these rules to mimic regularities observed in the foreign exchange markets.

The remainder of the paper is organized as follows. In section two, we develop the baseline model of the exchange rate and we specify the way agents form their expectations about the future exchange rate. In section three, we introduce the learning

\footnotetext{
${ }^{1}$ See Sarno and Taylor(2002), De Grauwe and Grimaldi(2006).
} 
rules of the agents. In section four, we study the steady state properties of the models. Section five presents a numerical analysis of the dynamics of the exchange rate. We carry out sensitivity analysis of the two learning models in section six. Section seven confronts the statistical properties of the exchange rate under the two learning rules with the data. Section eight provides some concluding remarks.

\section{Exchange rate model and agents' expectations}

\subsection{Asset pricing model of the exchange rate}

We model the market exchange rate using an asset pricing view of the exchange rate. This allows us to write the exchange rate as:

$$
s_{t}=s_{t}^{*}+b\left(E_{t} s_{t+1}-s_{t}\right)
$$

where $s_{t}$ is the log level of the exchange rate in period $t$, defined as the domestic price of a unit of foreign currency and $s_{t}^{*}$ defines the set of fundamentals. Equation (1) expresses the market exchange rate as the sum of the current fundamentals and the expected change of the market. Model (1) can be viewed as the reduced form of the monetary model linking the exchange rate to money supplies and incomes. It can also correspond to the models of stock valuation, where $s_{t}^{*}$ plays a role of dividends and $b$ is a discount factor applied to expected future capital gains. We rewrite this equation in the following form:

$$
s_{t}=(1-\alpha) s_{t}^{*}+\alpha E_{t} s_{t+1}+\eta_{t}
$$

where $\alpha=\frac{b}{1+b}$, and $1-\alpha=\frac{1}{1+b}$. Thus, the market exchange rate is a convex combination of the fundamental rate and the expectations of the future market exchange rate. We assume that there are unexpected disturbances in the market process captured by $\eta_{t} \sim$ iid $\left(0, \sigma_{\eta}^{2}\right)$. Note that $\alpha$ can be interpreted as a discount factor on the future market exchange rate expectations and thus $0<\alpha<1$. We also assume that the $\log$ fundamental $s_{t}^{*}$ is driven by a random walk, i.e.

$$
s_{t}^{*}=s_{t-1}^{*}+\epsilon_{t}
$$


where $\epsilon_{t} \sim i i d\left(0, \sigma_{\epsilon}^{2}\right)$.

Solving Model (2) assuming rational expectations of agents yields:

$$
\begin{aligned}
s_{t}= & (1-\alpha) s_{t}^{*}+\alpha\left(E_{t}(1-\alpha) s_{t+1}^{*}+\alpha(1-\alpha) s_{t+2}^{*}+\alpha^{2}(1-\alpha) s_{t+3}^{*}\right. \\
& \left.+\ldots+\alpha^{n-1}(1-\alpha) s_{t+n}^{*}+\eta_{t+1}+\eta_{t+2}+\ldots+\eta_{t+n}\right)+\alpha^{n} E_{t} s_{t+n}+\eta_{t}
\end{aligned}
$$

Note that for stationarity of the above solution, we need $\alpha<1$. Using the definition of the fundamental process in equation (3), we find:

$$
\begin{aligned}
s_{t}= & (1-\alpha) s_{t}^{*}+\alpha\left((1-\alpha) s_{t}^{*}+\alpha(1-\alpha) s_{t}^{*}+\alpha^{2}(1-\alpha) s_{t}^{*}\right. \\
& \left.+\ldots+\alpha^{n-1}(1-\alpha) s_{t}^{*}\right)+\alpha^{n} E_{t} s_{t+n}+\eta_{t}
\end{aligned}
$$

Finally, letting $n \rightarrow \infty$ and assuming the absence of rational bubbles, namely that $\lim _{n \longrightarrow \infty} \alpha^{n} E_{t} s_{t+n}=0$, we find:

$$
s_{t}=(1-\alpha) s_{t}^{*}+\alpha s_{t}^{*}+\eta_{t}=s_{t}^{*}+\eta_{t}
$$

We find that under rational expectations assumption, the market exchange rate is driven by the current fundamental rate and some unexpected noise.

\subsection{The expectations formation}

In this section, we specify the mechanism determining expectations of agents and we depart from the assumption of rational expectations. We take the view that the rational expectations assumption puts too great an informational burden on individual agents. Agents experience cognitive problems in processing information. As a result, they use simple forecasting rules (heuristics). They are willing to learn however. Their learning process will then lead them to put different weights on the rules they are using.

We start by assuming that agents can use two different forecasting rules. One will be called a fundamentalist forecasting rule, the other a chartist rule (technical analysis). Thus we introduce heterogeneity in the agents' forecasts ${ }^{2}$.

\footnotetext{
${ }^{2}$ Survey data indicate that the expectations in the exchange market are not homogeneous (Taylor and Allen (1992), Frankel and Froot (1990), Bénassy-Quéré, Larribeau and Macdonald (1999)). These survey data point out that the FOREX traders do not stick to one single trading rule. They alter and even mix the trading rules according to the realized profits.
} 
When using a fundamentalists rule, agents compare the market exchange rate with the fundamental rate and they forecast the future market rate to return to the fundamental rate:

$$
E_{t}^{f}\left(\tilde{\Delta} s_{t+1}\right)=-\psi\left(s_{t-1}-s_{t-1}^{*}\right)
$$

where $\tilde{\Delta} s_{t+1}$ is defined as $s_{t+1}-s_{t-1}$. We assume here that boundedly rational agents do not know $s_{t}$ at $t$. We can rewrite also the expectations for $s_{t+1}$ :

$$
E_{t}^{f}\left(s_{t+1}\right)=s_{t-1}+\psi\left(s_{t-1}^{*}-s_{t-1}\right)
$$

In this sense, they follow a negative feedback rule: where $\psi>0$ is a parameter describing the speed at which the agents expect the exchange rate to return to its fundamental value.

The second forecasting rule agents use is a chartist rule. We assume that this takes the form of extrapolating the last change of the exchange rate into the future:

$$
E_{t}^{c}\left(\tilde{\Delta} s_{t+1}\right)=\beta \Delta s_{t-1}
$$

where $\Delta s_{t-1}=s_{t-1}-s_{t-2}$. Alternatively we can write:

$$
E_{t}^{c}\left(s_{t+1}\right)=s_{t-1}+\beta \Delta s_{t-1}
$$

The degree of extrapolation is given by the parameter $\beta>0$. Clearly, more sophisticated rules could be specified. Here we focus on the simplest possible chartist rule.

The agents combine these two rules with their respective weights. As a result, the market forecast, $E_{t} s_{t+1}$, is assumed to be a weighted average of the mean-reverting and the extrapolative components:

$$
E_{t} s_{t+1}=\omega^{f} E_{t}^{f} s_{t+1}+\omega^{c} E_{t}^{c} s_{t+1}
$$

where $E_{t}^{f} s_{t+1}$ and $E_{t}^{c} s_{t+1}$ are the mean-reverting (fundamentalist) and the extrapolative (chartist) components, respectively, $\omega^{f}$ is the weight given to the fundamentalist rule, $\omega^{c}$ is the weight given to the chartist rule and $\omega^{f}+\omega^{c}=1$.

The timing in this model should be specified carefully. Since our agents are boundedly rational, they do not know the current exchange rate that will be the outcome of their forecast. The last available information they have about the exchange rate is the one 
prevailing in the previous period. Thus, when they make a forecast in period $t$ they use the information up to period $t-1$.

We now substitute equation (8) and (10) into equation (11) and the latter into equation (2). This yields:

$$
s_{t}=(1-\alpha) s_{t}^{*}+\alpha \omega^{f}\left[s_{t-1}+\psi\left(s_{t-1}^{*}-s_{t-1}\right)\right]+\alpha \omega^{c}\left[s_{t-1}+\beta \Delta s_{t-1}\right]+\eta_{t}
$$

\section{Learning mechanisms of agents}

In our world of bounded rationality, agents use simple rules described in the previous section. However, they test these rules continuously. This testing procedure is the mechanism by which discipline is imposed on the behavior of individual agents. We specify two alternative testing procedures (learning mechanisms). In the first one, agents select the rules based on a fitness method. In the second mechanism, agents learn to improve these rules using statistical methods. The main difference between both learning strategies lies in the assumption which parameters are time-varying. In the fitness learning, the market expectations change because of the shifts of the weights on two rules, while the parameters $\psi$ and $\beta$ are fixed. In the statistical learning, the opposite takes place. The weights on two rules are equal and constant, and the agents estimate the parameters of the rules $\psi$ and $\beta$.

\subsection{Fitness mechanism}

The first learning mechanism is based on a fitness criterion in the spirit of Brock and Hommes (1997), (1998), which is based on discrete choice theory ${ }^{3}$. This mechanism assumes that agents evaluate the two forecasting rules by computing their past profitability and to increase (reduce) the weight of one rule if it is more (less) profitable than the alternative rule. We specify this procedure as follows:

\footnotetext{
${ }^{3}$ This specification is often applied in discrete choice models. For an application in the markets for differentiated goods, see Anderson, et al., (1992). There are other ways to specify a rule that governs the selection of forecasting strategies. One was proposed by Kirman(1993). Another one was formulated by Lux and Marchesi(1999).
} 


$$
\begin{aligned}
\omega_{t}^{f} & =\frac{\exp \delta \pi_{t}^{f \prime}}{\exp \delta \pi_{t}^{f \prime}+\exp \delta \pi_{t}^{c \prime}} \\
\omega_{t}^{c} & =\frac{\exp \delta \pi_{t}^{c \prime}}{\exp \delta \pi_{t}^{c \prime}+\exp \delta \pi_{t}^{f \prime}}
\end{aligned}
$$

where $\omega_{t}^{f}$ and $\omega_{t}^{c}$ are the weights given to the fundamentalist and chartist rules, respectively. $\pi_{t}^{f \prime}$ and $\pi_{t}^{c \prime}$ are the (risk adjusted) profits. These are defined as:

$$
\pi_{t}^{f \prime}=\pi_{t}^{f}-\mu \sigma_{f, t}^{2}
$$

and

$$
\pi_{t}^{c \prime}=\pi_{t}^{c}-\mu \sigma_{c, t}^{2}
$$

where $\mu$ is the coefficient of risk aversion and $\pi_{t}^{f}$ and $\pi_{t}^{c}$ are the profits made in forecasting, while $\sigma_{f, t}^{2}$ and $\sigma_{c, t}^{2}$ are the variances of the forecast errors made using fundamentalist and chartist rules, respectively.

We define the profits of fundamentalists and chartists rules, $\pi_{t}^{f}$ and $\pi_{t}^{c}$, respectively, as the one-period returns of investing in the foreign asset:

$$
\begin{gathered}
\pi_{t}^{i}=\left(s_{t-1}-s_{t-2}\right) \operatorname{sgn}\left(E_{t-1}^{i} s_{t-1}-s_{t-2}\right) \\
\text { where } \operatorname{sgn}[x]=\left\{\begin{array}{cl}
1 & \text { for } x>0 \\
0 & \text { for } x=0 \\
-1 & \text { for } x<0
\end{array} \quad \text { and } i=c, f\right.
\end{gathered}
$$

Thus, when agents forecasted an increase in the exchange rate return (the return of the foreign currency) and this increase is realized, their profit is equal to the observed increase in the exchange rate return. If instead the exchange rate return declines, they make a loss which equals this decline (because in this case they have bought foreign assets which have declined in return).

Equations (13) and (14) can now be interpreted as follows. When the risk adjusted profits of the extrapolative (chartist) rule increase, relative to the risk adjusted profits of the mean-reverting (fundamentalist) rule, then the weight the agents give to the extrapolative rule in period $t$ increases, and vice versa. The parameter $\delta$ measures the intensity with which the agents switch the weights from one rule to the other. With 
an increasing $\delta$ agents react strongly to the relative profitability of the two forecasting rules. In the limit, when $\delta$ goes to infinity, the agents choose the forecasting rule which proves to be more profitable. When $\delta$ is equal to zero, agents are insensitive to the relative profitability of these rules. In the latter case, the weights of mean-reverting and extrapolative rules are constant and equal to 0.5. Thus, $\delta$ is a measure of inertia in the decision to give more weight to the more profitable rule ${ }^{4}$.

The weights obtained from equations (13) and (14) are then substituted into the rate equation(12):

$$
\begin{gathered}
s_{t}=(1-\alpha) s_{t}^{*}+\alpha\left[\omega_{t}^{f} E_{t}^{f}\left(s_{t+1}\right)+\omega_{t}^{c} E_{t}^{c}\left(s_{t+1}\right)\right]+\eta_{t} \\
E_{t}^{f}\left(s_{t+1}\right)=s_{t-1}+\psi\left(s_{t-1}^{*}-s_{t-1}\right) \\
E_{t}^{c}\left(s_{t+1}\right)=s_{t-1}+\beta \Delta s_{t-1}
\end{gathered}
$$

Note that in this learning mechanism agents are assumed to use the same values of parameters $\beta$ and $\psi$ in every period $t$. However, they give different weights to these parameters each period, depending on how well the forecasting rules underlying these parameters do in terms of profitability.

\subsection{Statistical learning}

The second learning mechanism that we consider here is statistical learning (see Evans and Honkapoja (2001)). As before, agents' expectations are composed of two components, i.e. a mean-reverting and an extrapolative one. Agents are assumed to have some basic knowledge of econometrics and they estimate the importance of these two components based on data up to period $t-1$. Their expectations are formed in the following way:

$$
E_{t}\left(\tilde{\Delta} s_{t+1}\right)=\psi_{t-1}\left(s_{t-1}^{*}-s_{t-1}\right)+\beta_{t-1} \Delta s_{t-1}
$$

\footnotetext{
${ }^{4}$ The logic of the switching weight is the same spirit of the adaptive rules that are used in game theoretic models (See, for examples, Cheung and Friedman (1997); Fudenberg and Levine, (1998)). In these models, actions that did better in the observed past tend to increase in frequency while actions that did worse tend to decrease in frequency.
} 
At time $t+1$, as the realized values of the market $\left(s_{t}\right)$ and fundamental $\left(s_{t}^{*}\right)$ exchange rates are available, agents revise their forecasting rule. In particular, according to their forecasting rule, they regress $\Delta s_{t}$ on $s_{t-1}^{*}-s_{t-1}$ and $\Delta s_{t-1}$. They update the parameters $\psi_{t-1}$ and $\beta_{t-1}$ using recursive methods ${ }^{5}$ and the last available information, i.e. $s_{t}$ :

$$
\begin{aligned}
\phi_{t} & =\phi_{t-1}+\gamma_{t} R_{t}^{-1} z_{t-1}\left(\Delta s_{t}-\phi_{t-1}^{\prime} z_{t-1}\right) \\
R_{t} & =R_{t-1}+\gamma_{t}\left(z_{t-1} z_{t-1}^{\prime}-R_{t-1}\right)
\end{aligned}
$$

where $\phi_{t}=\left(\psi_{t}, \beta_{t}\right)^{\prime}$ is the vector of parameter estimates, $z_{t-1}=\left(s_{t-1}^{*}-s_{t-1}, \Delta s_{t-1}\right)$ is a vector of explanatory variables, $R_{t}=\gamma_{t} \sum_{i=1}^{t} z_{i-1} z_{i-1}^{\prime}$ is a moment matrix and $\gamma_{t}$ is the gain. The gain captures the speed of updating in the sense of how much weight the agents put on the new incoming information. We will assume that agents put more weight on new information and thus update their forecasts with a low constant gain. Introducing the perceived law of motion (PLM) as given by equation (21) into equation (2), we obtain the resulting actual law of motion (ALM) of the market exchange rate:

$$
s_{t}=(1-\alpha) s_{t}^{*}+\alpha\left(1+\beta_{t-1}-\psi_{t-1}\right) s_{t-1}-\alpha \beta_{t-1} s_{t-2}+\alpha \psi_{t-1} s_{t-1}^{*}+\eta_{t}
$$

\section{Steady state properties}

In this section, we analyze the steady state properties of the market exchange rate under two learning mechanisms. This will allow us to analyze the question of whether these two learning mechanisms are capable of revealing the fundamental value of the exchange rate in the steady state.

\subsection{The steady state under fitness learning}

In order to analyze the steady state of the model under fitness mechanism, we strip it from its stochastic components. Thus we assume that the fundamental variable is

\footnotetext{
${ }^{5}$ Note that $\psi_{t-1}$ should now be interpreted as time varying expression $\omega^{f} \psi$ used in the previous section and $\beta_{t-1}$ as $\omega^{c} \beta$ where $\omega^{f}=\omega^{c}=0.5$.
} 
constant. In addition, for the sake of convenience, we set the fundamental rate, $s_{t}^{*}=$ $s^{*}=0$. As a result, the exchange rate movements can be interpreted as deviations from their fundamental value.

We rewrite equation (18) for as follows:

$$
s_{t}=\alpha\left[s_{t-1}-\omega_{t-1}^{f} \psi s_{t-1}+\left(1-\omega_{t-1}^{f}\right) \beta\left(s_{t-1}-s_{t-2}\right)\right]
$$

where

$$
\omega_{t-1}^{f}=\frac{\exp \left[\gamma\left(\pi_{t-1}^{f}-\mu \sigma_{f, t-1}^{2}\right)\right]}{\exp \left[\gamma\left(\pi_{t-1}^{c}-\mu \sigma_{c, t-1}^{2}\right)\right]+\exp \left[\gamma\left(\pi_{t-1}^{f}-\mu \sigma_{f, t-1}^{2}\right)\right]}
$$

Variance terms from equations (15) and (16) can be written as follows:

$$
\begin{aligned}
\sigma_{c, t-1}^{2} & =\left[E_{t-2}^{c}\left(s_{t-1}\right)-s_{t-1}\right]^{2} \\
\sigma_{f, t-1}^{2} & =\left[E_{t-2}^{f}\left(s_{t-1}\right)-s_{t-1}\right]^{2}
\end{aligned}
$$

Using the definition of the forecasting rules (8) and (10) this yields

$$
\begin{gathered}
\sigma_{c, t-1}^{2}=\left[-\beta s_{t-3}+(1+\beta) s_{t-2}-s_{t-1}\right]^{2} \\
\sigma_{f, t-1}^{2}=\left[(1-\psi) s_{t-2}-s_{t-1}\right]^{2}
\end{gathered}
$$

With suitable changes of variables it is possible to write these equations as a 3dimensional system. Set:

$$
\begin{gathered}
u_{t}=s_{t-1} \\
x_{t}=u_{t-1}\left(=s_{t-2}\right)
\end{gathered}
$$

The 3 dynamic variables are $\left(s_{t}, u_{t}, x_{t}\right)$. The state of the system at time $t-1$, i.e. $\left(s_{t-1}, u_{t-1}, x_{t-1}\right)$ determines the state of the system at time $t$, i.e. $\left(s_{t}, u_{t}, x_{t}\right)$ through the following $3-D$ dynamical system:

$$
s_{t}=\alpha\left[\left(1-\omega_{t-1}^{f}\right)\left((\psi+\beta) u_{t}-\beta x_{t}\right)\right]
$$




$$
\begin{aligned}
& u_{t}=s_{t-1} \\
& x_{t}=u_{t-1}
\end{aligned}
$$

where $\omega_{t-1}^{f}$ is defined in equation (25) and the forecast errors and ex-post profits are defined in following way:

$$
\begin{gathered}
\sigma_{c, t-1}^{2}=\left[(1+\beta) u_{t-1}-\beta x_{t-1}-s_{t-1}\right]^{2} \\
\sigma_{f, t-1}^{2}=\left[(1-\psi) u_{t-1}-s_{t-1}\right]^{2} \\
\pi_{t-1}^{c}=\left(s_{t-1}-u_{t-1}\right) \operatorname{sgn}\left[\beta\left(u_{t-1}-x_{t-1}\right)\right] \\
\pi_{t-1}^{f}=\left(s_{t-1}-u_{t-1}\right) \operatorname{sgn}\left[-\psi u_{t-1}\right]
\end{gathered}
$$

We can now analyze the nature of the steady state solution. Since we have normalized the fundamental exchange rate $s_{t}^{*}$ to be zero, the fundamental solution implies that $s_{t}=0$. As a result, the variance terms go to zero.

The steady state of the system is now obtained by setting:

$$
\left(s_{t-1}, u_{t-1}, x_{t-1}\right)=\left(s_{t}, u_{t}, x_{t}\right)=(\bar{s}, \bar{u}, \bar{x})
$$

in the dynamical system (30)-(32).

There is a unique steady state where

$$
\bar{s}, \bar{u}, \bar{x}=0
$$

Notice also that at the steady state:

$$
\bar{s}=\bar{u}=\bar{x}=s^{*}, \bar{\omega}^{c}=\frac{1}{2}, \bar{\omega}^{f}=\frac{1}{2}, \bar{\pi}^{f}=\bar{\pi}^{c}=0, \bar{\sigma}_{f}^{2}=\bar{\sigma}_{c}^{2}=0
$$

i.e. the steady state is characterized by the exchange rate being at its fundamental level, by zero profits and zero risk, and by fundamentalist and technical trader fractions equal to $\frac{1}{2}$. 
We can also analyze the conditions under which a non-zero steady state solution exists. This is a solution in which the exchange rate is constant and permanently different from its (constant) fundamental value. If such a second steady state solution exists, the model allows for an exchange rate in the steady state that is permanently disconnected from its fundamental.

In order to analyze under what condition such a steady state solution can arise, we use equation (2) and set $s_{t}=s_{t-1}=s_{t-2}=\bar{s}$, so that

$$
\bar{s}=\alpha\left(1-\omega_{t}^{f} \psi\right) \bar{s}
$$

It can now easily be seen that a solution of the type $\bar{s} \neq 0$ exists iff $\alpha\left(1-\omega_{t}^{f} \psi\right)=$ 1. This condition is satisfied if $\alpha=1$ and $\omega_{t}^{f}=0$. The first of these two conditions says that the current fundamental should have no influence on the current exchange rate; the second condition says that the share of the fundamentalists in the market should be zero. The latter, however, can only arise if $\sigma_{f, t}^{2} \rightarrow \infty$ (This can be seen from the definition of $\omega_{t}^{f}$ in (25)). As a result, a solution whereby the market exchange rate permanently deviates from the market exchange rate can be ruled out.

We conclude that in the steady state, the exchange rate equals its fundamental value and the learning based on the fitness method reveals the fundamental value.

\subsection{The steady state under statistical learning}

In this subsection, we analyze the properties of the steady state of the model under statistical learning. For the sake of simplicity, we assume that the agents learn using decreasing gain i.e., simple Least Squares updating. The agents' PLM is of the following form:

$$
\tilde{\Delta} s_{t+1}=\psi_{t-1}\left(s_{t-1}^{*}-s_{t-1}\right)+\beta_{t-1} \Delta s_{t-1}+\varsigma_{t+1}
$$

Accordingly, the agents form their expectations:

$$
E_{t}\left(\tilde{\Delta} s_{t+1}\right)=\psi_{t-1}\left(s_{t-1}^{*}-s_{t-1}\right)+\beta_{t-1} \Delta s_{t-1}
$$

where they forecast the market exchange rate return $\tilde{\Delta} s_{t+1}$. We can write equation (38): 


$$
E_{t} s_{t+1}=s_{t-1}+\psi_{t-1}\left(s_{t-1}^{*}-s_{t-1}\right)+\beta_{t-1} \Delta s_{t-1}
$$

Substituting the PLM into equation (2), yields the resulting ALM of the market exchange rate:

$$
s_{t}=(1-\alpha) s_{t}^{*}+\alpha(1+\beta-\psi) s_{t-1}-\alpha \beta s_{t-2}+\alpha \psi s_{t-1}^{*}+\eta_{t}
$$

Using the ALM for $s_{t-1}$ and the definition of the fundamental rate $s_{t}^{*}$ in equation (3), we obtain the following specification of the market exchange rate:

$$
\begin{aligned}
s_{t} & =[(1-\alpha)+\alpha \psi+\alpha(1+\beta-\psi)(1-\alpha)] s_{t-2}^{*}+ \\
& {\left[\alpha^{2}(1+\beta-\psi)-\alpha \beta\right] s_{t-2}+\alpha^{2} \beta(1+\beta-\psi)\left(s_{t-2}-s_{t-3}\right)+} \\
& \alpha^{2} \psi(1+\beta-\psi)\left(s_{t-2}^{*}-s_{t-2}\right)+[(1-\alpha)+\alpha \psi+\alpha(1+\beta-\psi)(1-\alpha)] \epsilon_{t-1}+ \\
& (1-\alpha) \epsilon_{t}+[\alpha(1+\beta-\psi)(1-\alpha)] \eta_{t-1}+\eta_{t}
\end{aligned}
$$

After substraction $s_{t-2}$ from both sides and carrying out some manipulations, we can rewrite the ALM in the same form as the PLM (equation (21)):

$$
\begin{aligned}
& s_{t}-s_{t-2}=(\alpha \psi-\alpha+1)(\alpha+\alpha \beta-\alpha \psi+1)\left(s_{t-2}^{*}-s_{t-2}\right)+ \\
& \alpha^{2}(1+\beta-\psi) \beta\left(s_{t-2}-s_{t-3}\right)+[(1-\alpha)+\alpha \psi+\alpha(1+\beta-\psi)(1-\alpha)] \epsilon_{t-1}+ \\
& (1-\alpha) \epsilon_{t}+[\alpha(1+\beta-\psi)(1-\alpha)] \eta_{t-1}+\eta_{t}
\end{aligned}
$$

This allows us to define T-map as labeled by Evans and Honkapohja (2001):

$$
T\left(\begin{array}{l}
\psi \\
\beta
\end{array}\right)=\left(\begin{array}{l}
(\alpha \psi-\alpha+1)(\alpha+\alpha \beta-\alpha \psi+1) \\
\alpha^{2}(1+\beta-\psi) \beta
\end{array}\right)
$$

From the system(44), we can compute the stationary points of $T(\psi, \beta)^{\prime}$. From the second equation of this system, we obtain two solutions for $\beta$ i.e., $\beta_{1}=0$ or $\beta_{2}=-1+\psi+\frac{1}{\alpha^{2}}$. We calculate the resulting solutions for $\psi$, for each of the fixed points of $\beta$. When $\beta_{1}=0$, we have two possible solutions for $\psi_{1}=1$ or $\psi_{2}=1-\frac{1}{\alpha^{2}}$. For $\beta_{2}=-1+\psi+\frac{1}{\alpha^{2}}$, we find $\psi_{3}=1-\frac{1}{\alpha^{2}}$. Substituting this result in $\beta_{2}$, this yields $\beta_{2}=0$. As a result, we have two possible pairs of solutions. The first one is given by the combination $\phi_{1}=\left(\begin{array}{c}\psi_{1} \\ \beta_{1}\end{array}\right)$ and means that the agents learn that the extrapolating component does not play a role in determination of the market exchange rate $\left(\beta_{1}=0\right)$. They find 
that the market exchange rate will return to the fundamental rate in the next period $\left(\psi_{1}=1\right)$. Substituting these values into the ALM (41), we obtain :

$$
s_{t}=s_{t}^{*}+\eta_{t}-\alpha \epsilon_{t}
$$

Thus, we find that this set of fixed points leads to the near rational expectations solution of the model ${ }^{6}$. Equation (45) includes an additional element with respect to the REE described by equation (6), namely $\alpha \epsilon_{t}$. The existence of this noise term in the equilibrium exchange rate process is due to the different assumptions about the information set the agents use. Rational agents are assumed to know the fundamental value of the exchange rate $s_{t}^{*}$ at $t$, while the agents who use statistical methods do not have $s_{t}^{*}$ in their information set at $t$. As a result, these agents face an additional uncertainty about the exchange rate, reflected by $\alpha \epsilon_{t}$. In the steady state, assuming for the sake of simplicity, $s_{t}^{*}=s_{t-1}^{*}=\bar{s}^{*}=0$ and $\eta_{t}=\epsilon_{t}=0$, we find $s_{t}=0$. This means that in the steady state the adaptive learning model leads the exchange rate to its fundamental value. The second pair of steady state solutions is given by $\phi_{2}=\left(\begin{array}{l}\psi_{2} \\ \beta_{2}\end{array}\right)$ and indicates that the agents again learn that extrapolating parameter to be zero $\left(\beta_{2}=0\right)$ and a negative value of $\psi_{2}$. This means that the fundamentalists learn to extrapolate the difference between market and fundamental exchange rates. We substitute the values of the second solution i.e., $\psi_{2}=1-\frac{1}{\alpha^{2}}$ and $\beta_{2}=0$ into the ALM (41). We find that the current market exchange rate is a sum of the fundamental rate and the extrapolated difference between past market and fundamental rates:

$$
s_{t}=s_{t}^{*}+\frac{1}{\alpha}\left(s_{t-1}-s_{t-1}^{*}\right)-\alpha v_{t}+\eta_{t}
$$

If we again assume that in the steady state $s_{t}^{*}=s_{t-1}^{*}=\bar{s}^{*}=0$ and $\epsilon_{t}, \eta_{t}=0$, we find that $s_{t}=0$. We conclude that the only existing equilibrium is the one when the market exchange rate equals the fundamental rate. The two solutions however imply different short run dynamics. The first set of fixed points $\phi_{1}$, leading to near rational expectations solution implies that the market rate is permanently connected to the fundamental rate. The second solution $\phi_{2}$, allows for some short run disconnection from the fundamental

\footnotetext{
${ }^{6}$ We call the solution near rational expectations when it depends on the same state variables that REE and differs from it only in iid error term.
} 
rate. We study the short dynamics of the market rate within the proposed model in the following section.

We check the expectational stability (E-stability) of the two possible solutions by calculating the eigenvalues of $D T-I \lambda$. First, we compute the Jacobian matrix $D T\left(\begin{array}{l}\psi \\ \beta\end{array}\right)$ :

$$
D T\left(\begin{array}{l}
\psi \\
\beta
\end{array}\right)=\left(\begin{array}{ll}
\alpha^{2} \beta & \alpha(\alpha \psi-\alpha+1) \\
-\alpha^{2} \beta & \alpha^{2}(1+2 \beta-\psi)
\end{array}\right)
$$

We calculate the eigenvalues of the matrix $D T-I \lambda$ :

$$
\begin{gathered}
D T-I \lambda=\left(\begin{array}{cc}
\alpha^{2} \beta-\lambda & \alpha(\alpha \psi-\alpha+1) \\
-\alpha^{2} \beta & \alpha^{2}(1+2 \beta-\psi)-\lambda
\end{array}\right) \\
\left.\operatorname{det}(D T-I \lambda)=\left(\alpha^{2} \beta-\lambda\right)\left(\alpha^{2}(1+2 \beta-\psi)-\lambda\right)\right)+\alpha^{3} \beta(\alpha \psi-\alpha+1)=0
\end{gathered}
$$

Hence, we find the solution for $\lambda$ :

$$
\lambda=\frac{\alpha^{2}(1+2 \beta-\psi) \pm \sqrt{\alpha^{4}(1+2 \beta-\psi)^{2}-4 \alpha^{3} \beta(\alpha(1+2 \beta-\psi)+(\alpha \psi-\alpha+1))}}{2}
$$

We now substitute into equation (50) two possible sets of solutions $\phi_{1}=\left(\begin{array}{c}\psi_{1} \\ \beta_{1}\end{array}\right)$ and $\phi_{2}=\left(\begin{array}{l}\psi_{2} \\ \beta_{2}\end{array}\right)$. For the first set of solutions $\phi_{1}=\left(\begin{array}{l}1 \\ 0\end{array}\right)$, we find that both eigenvalues are equal to zero and thus the solution $\phi_{1}$, leading to the near rational expectations equilibrium, is E-stable. The second set of solutions: $\phi_{2}=\left(\begin{array}{l}1-\frac{1}{\alpha^{2}} \\ 0\end{array}\right)$ yields the eigenvalues : $\lambda_{1}=0$ and $\lambda_{2}=1$. Since one of the eigenvalues $\left(\lambda_{2}=1\right)$ is at the border of E-stability/unstability, the analysis requires higher order approximation and the use of center manifold techniques. This analysis is beyond the scope of this paper.

\section{Dynamic analysis}

From the previous analysis it follows that both learning models allow the exchange rate to converge to its fundamental value. Thus, both learning mechanisms are efficient in revealing the fundamental value of the exchange rate in the steady state. These steady state properties, however, do not guarantee the same dynamic properties. As a result, these two learning mechanisms could produce very different short-term behavior 
of the exchange rate. The non-linear properties of the model make it very difficult to describe its dynamics with analytical tools. We therefore use numerical methods to analyse the dynamic properties of the model. We study these properties within two different cases. In the first case, we assume the values of the parameters to be such that they correspond to low frequency (quarterly) observations. In the second case, we assume them to correspond to high frequency (daily) observations. In these two cases, the discount factor $\alpha$ is calculated based on the assumption that the nominal interest rate $r=5 \%$ per year. We also calibrate the size of shocks. We assume that the news in fundamentals, $\epsilon_{t}$, and in the noise in the foreign exchange market, $\eta_{t}$, are of the same magnitude (their standard deviations are equal). The values of the standard deviations of these shocks are calculated on the basis of a simple fundamental model of the exchange rate which is studied in more detail in Section 7.

\subsection{Low frequency observations}

We show the contrast in the dynamics produced by the two learning mechanisms by simulating the model in the time domain over 10000 periods with a quarterly discount factor $\alpha=0.988\left(\alpha=\frac{1}{(1+0.05)^{\frac{1}{4}}}=0.988\right)$. The standard deviations of the fundamental shock and the market noise are equal $\sigma_{\epsilon}=\sigma_{\eta}$ and set to 0.03 . This number is obtained from the rescaled monthly standard deviation of the fundamental shock, found in the data and equal to 0.015 (see section 7 ). We calculate the quarterly equivalent by multiplying this number by $\sqrt{4}$. In the fitness learning model, we set the value of the parameter $\beta$ at 0.95 and $\psi$ at 0.25 . This choice may seem arbitrary but it is consistent with empirical evidence suggesting that, in the short run, agents expect the past change to be almost entirely extrapolated into the future, while they believe that, in the longer run, the market rate will return to the fundamental value ${ }^{7}$. The value of $\psi=0.25$ for quarterly observations suggests that the agents expect the market rate to return to the fundamental value in one year ${ }^{8}$.

\footnotetext{
${ }^{7}$ For the detailed description of the traders' forecasts see Cheung and Chinn (2001) and Cheung et al. (2004).

${ }^{8}$ The empirical evidence suggests that the convergence of market rate to the fundamental rate may take longer than one year. Frankel and Froot (1987) find an expected half-life for deviations from the fundamental proxied by PPP of around 3 years. Similarly, Mark (1995) and Chinn and Meese (1995) demonstrate that the models incorporating a set of fundamentals have some statistically significant power over the horizon of 3 years. These results are not inconsistent with traders' expectations. In fact, traders use both rules, i.e. fundamentalists and chartists forecasting rules, simaultaneously. The
} 
In the statistical learning model, we assume that the agents learn the underlying process of the market exchange rate using a constant gain $\gamma$. This assumption implies that traders put a higher weight on the more recent data. However, under the constant gain learning, the estimated coefficients do not converge to a single point, as suggested by the steady state analysis from the previous section, but to a distribution centered around the estimates ${ }^{9}$. As a result, we expect that the estimated coefficients will be more volatile, with the means corresponding to the solutions $\phi_{1}$. As a benchmark constant gain, we use the value estimated by F. Milani (2005), who estimated a constant gain used by the agents forming expectations about inflation. He found that agents make use of 13-14 years of data $(\gamma=0.0183)$. In Section 7 , we set a higher value of the gain, thus assuming faster learning.

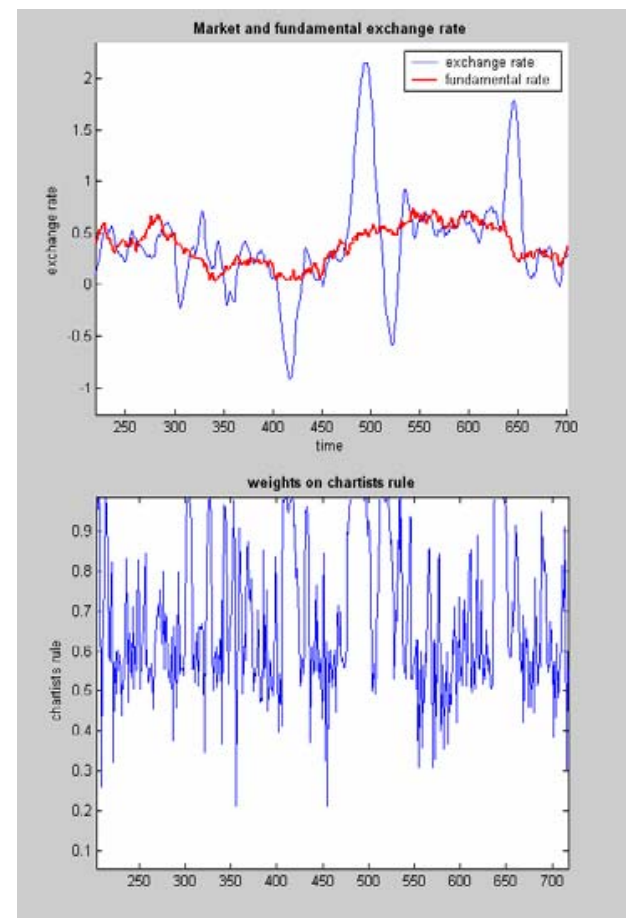

Figure 1: Exchange rate at low frequency under fitness learning and weight on chartists' rule

We present the results of fitness learning in Figure 1. The upper panel shows the

extrapolative behaviour of agents prevents the market rate from reaching its fundamental value over the horizon implied by the fundamentalists rule (Cheung et al. (2001)).

${ }^{9}$ See Evans and Honkapohja (2001) for the necessary conditions for the converegence of the constant gain algorithms. 
market and the fundamental exchange rates in the time domain for a subsample of 500 periods. We find that the market exchange rate is often disconnected from the fundamental one. As can be seen in Figure 1, the market exchange rate moves around the fundamental in a cyclical way. These cyclical movements have the appearance of bubbles and crashes. A comparison of the upper and lower parts of the left panel of Figure 1 allows us to understand the nature of these cycical movements. The lower part shows the weights on the chartists' rule. We find that periods of sustained deviations of the exchange rate from the fundamental coincide with periods during which the chartists' rule dominates the market expectations. We have analyzed this feature in the framework of a similar model in De Grauwe and Grimaldi (2005 and 2006).

Our interpretation of this result is that a series of stochastic shocks in one direction can lead to an increased profitability of the extrapolative (chartist) forecasting rule thereby leading to an increased popularity of this rule at the expense of the fundamentalist rule. This creates a self-fulfilling dynamics. As chartist rules become more profitable they get more weight in the market forecast, thereby intensifying the upward (downward) movement. At some point, however, movements in the fundamental have the effect of pulling the exchange rate back to its fundamental. We will return to this feature later and apply a sensitivity analysis to check under what conditions this dynamics occurs.

The results of statistical learning, shown in Figure 2, lead to a different conclusion. The difference between market and fundamental rates is very small. The mean difference is equal to -0.0012 in the sample shown in Figure 2. Thus, there does not seem to be disconnection as the exchange rate closely follows the fundamental rate. The lower panel of Figure 2 shows the parameters $\psi$ and $\beta$ estimated by the agents. As expected, they are very volatile and they fluctuate around 1 and 0 . Thus, the agents learn the values of parameters $\psi$ and $\beta$ distributed around the means leading to the fundamental exchange rate (see equation (45)). As a result, the market exchange rate is connected to the fundamental rate. 


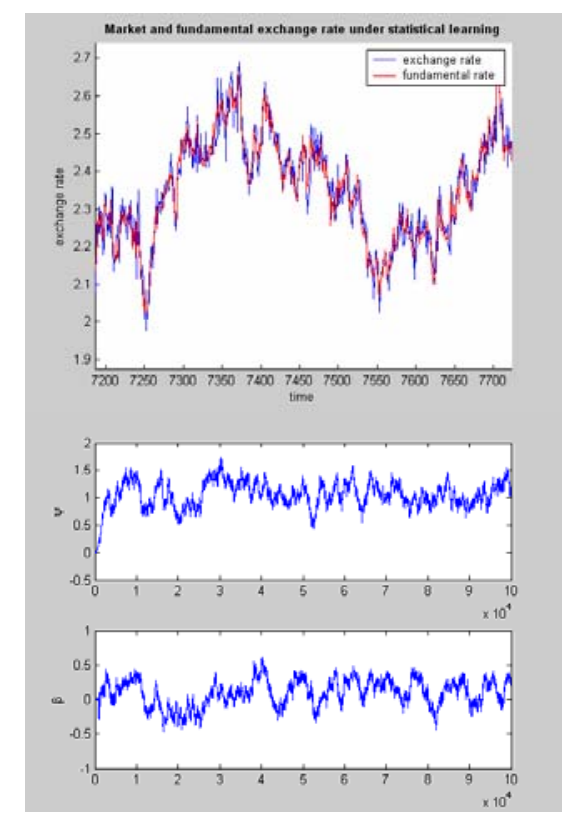

Figure 2: Exchange rate at low frequency under statistical learning and estimated parameters

\subsection{High frequency observations}

In this subsection, we analyze the model in an environment of high frequency observations. We calculate the daily discount factor $\alpha=\frac{1}{(1+0.05)^{\frac{1}{250}}}=0.999$ and the size of shocks: $\sigma_{v}=\sigma_{\eta}=\frac{0.015}{\sqrt{21}}=0.0033$.

In the fitness learning model we now set the parameter $\psi=0.004$ which is consistent with the assumption used earlier that the agents expect the market rate to converge to the fundamental rate in one year. We show an example of a simulation in the time domain in Figure 3. Note that we show the results of more periods than in the previous section, since they now correspond to a much shorter time span. The results are similar to those reported in the previous subsection. The market exchange rate is often disconnected from the underlying fundamental.

For the statistical learning model, we assume again that agents use $13-14$ years of data implying $\gamma=0.0003$ for daily data. We calculated this number assuming 250 trading days in a year: $\gamma=\frac{1}{3250}$.

An example of a simulation with statistical learning is shown in Figure 4. The upper 


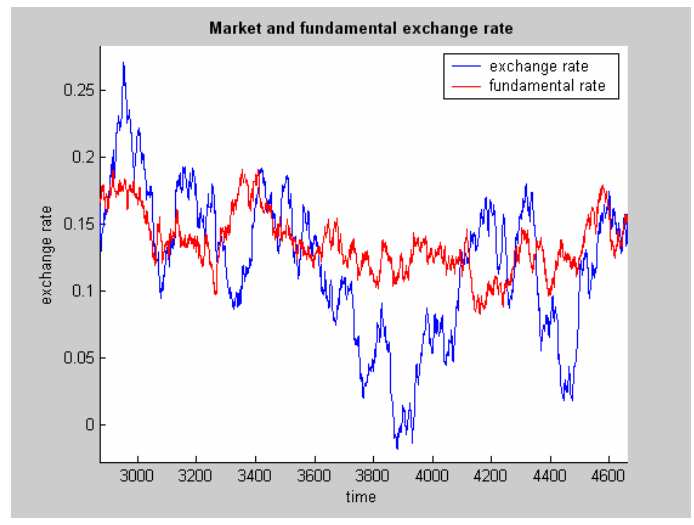

Figure 3: Exchange rate at high frequency under fitness learning and weight of chartists' rule

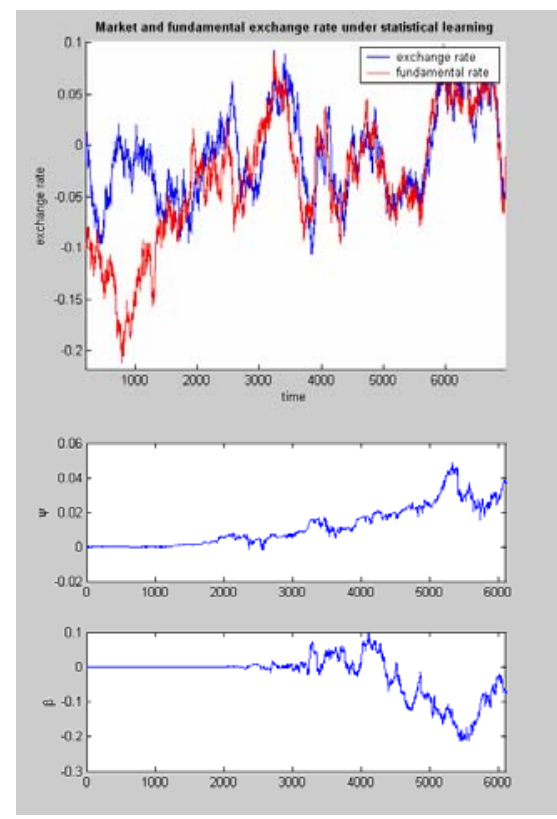

Figure 4: Exchange rate at high frequency under statistical learning and estimated parameters 
panel shows simulated market and fundamental rates and the lower panel presents the corresponding values of the parameters $\psi$ and $\beta$. Unlike the low frequency case, we find here that the market exchange rate can be disconnected from the fundamental value. Although the mean difference during the whole simulated sample is low, i.e. -0.0245 , we see in Figure 5 that this difference can even reach the numbers of the order of 0.2. The disconnection phenomenon is observed in the initial phase of the simulation period, however. After some time it tends to disappear as is made clear in Figure 5.

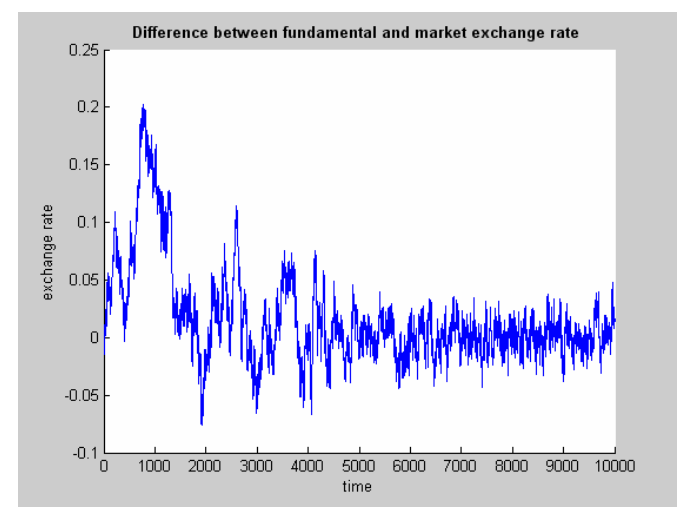

Figure 5: The difference between market and fundamental exchange rates

The disconnection between market and fundamental rates occurs because the agents are slow to learn the steady state values of the parameters $\psi$ and $\beta$ distributed around 1 and 0 , respectively. Thus statistical learning produces the disconnection phenomenon when applied to high frequency data, in particular, when the discount factor comes close to one (here $\alpha=0.999$ ). As we saw in Section 2, the REE is stationary, only when $\alpha<1$. Thus a value of $\alpha=1$ constitutes the border between stability and instability of the fundamental REE. When, in the stochastic environment, $\alpha$ gets closer to this border, agents are very slow to learn about the fundamental and the exchange rate can be attracted by unstable solutions. The intuition of this result is that in a high frequency environment, the attempt to update the estimated coefficients on a daily basis is likely to run into difficulties. The signal to noise ratio is very low. As a result, the agents need a great amount of data to find out that the fundamental influences the market exchange rate. This can also be seen in the lower panel of Figure 4 . In the 
beginning of the learning process, when the agents do not have a lot of observations, both parameters are very close to zero. As a result, the market rate is disconnected from the fundamental rate. When more data become available, agents find the parameters $\psi$ and $\beta$ to be different from zero. In particular, we observe that the parameter $\psi$ tends to increase. As a result, the difference between market and fundamental rates declines and the disconnection tends to disappear.

\section{$6 \quad$ Sensitivity analysis}

In the previous section, we found that both, fitness and statistical learning models can generate a market exchange rate disconnected from the fundamental rate. Fitness learning however can produce this disconnection in the high as well as in the low frequency cases, while under statistical learning the disconnection occurs only in the high frequency case when agents have not yet collected sufficient data to estimate the underlying parameters accurately. In this section, we analyze these characteristics in more detail by checking how sensitive these results are to different parameter values. In particular, we check under what conditions this disconnection emerges.

\subsection{Sensitivity to $\beta$ of the fitness learning model}

In order to better understand the cyclical nature of the short-term dynamics in the fitness learning, we performed a sensitivity analysis whereby we allowed the parameter $\beta$ and the initial conditions to change. We simulated the model assuming that the stochastic realization of the fundamental was identical for all the different values of the parameter $\beta$ and for all initial conditions. We then simulated the model for different time lengths going from $T=50$ to $T=1000$ and we collected the deviation of the exchange rate from its fundamental in period $T$. We show the results in Figure 6. On the vertical axis we set out the deviation of the exchange rate from its fundamental after 50,200,500and 1000 periods respectively. On the $\mathrm{x}$-axis we show the different values of the parameter $\beta$, and on the y-axis the different initial conditions. We find first that when the model is simulated over short periods of time, the exchange rate can deviate significantly from its fundamental. When for example the model is simulated for $T=50$, the exchange rate in period $\mathrm{T}$ deviates from its fundamental when initial 


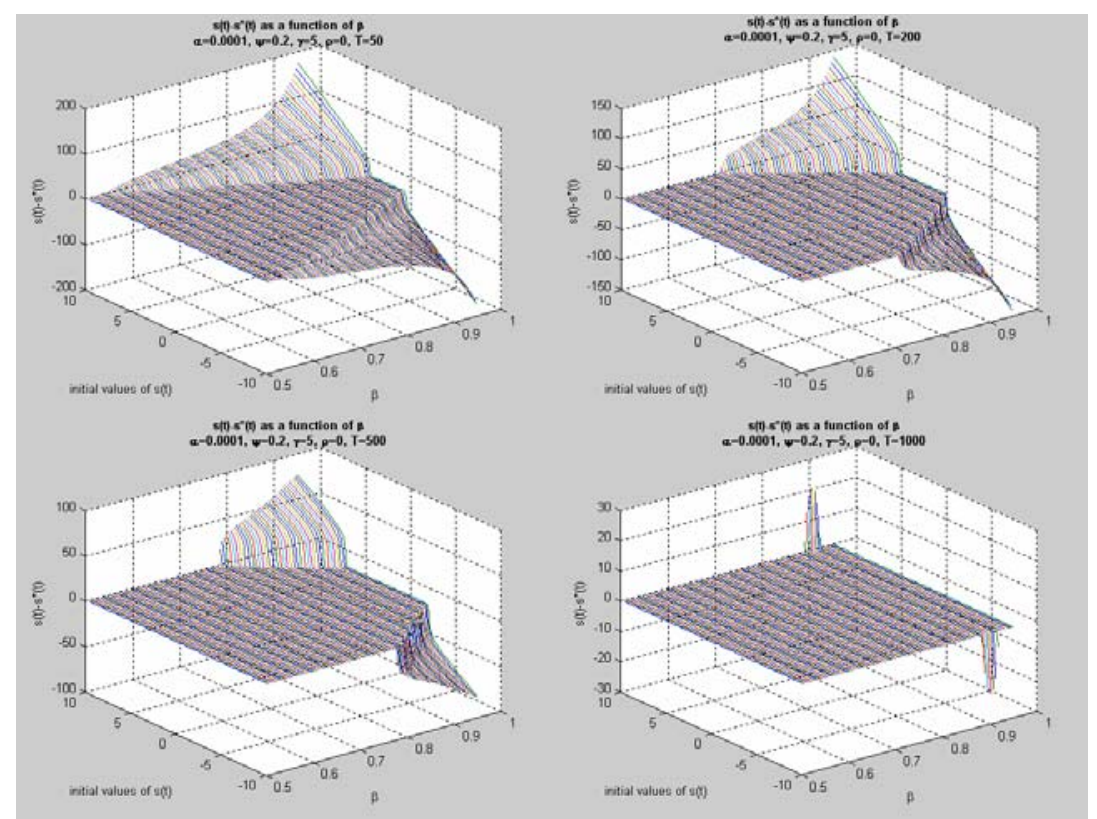

Figure 6: Difference between market and fundamental rates as a function of initial conditions and beta under fitness learning

shocks are sufficiently large and when $\beta$ increases. Thus, it appears that when the initial shock is large enough and when $\beta$ is large enough, the exchange rate remains disconnected from its fundamental value even after 50 periods. As the simulation period is extended, the area of disconnections shrinks. When $T$ exceeds 1000, the exchange rate has returned to its fundamental value for almost all initial conditions and for all values of $\beta$. This result is consistent with our steady state analysis which showed that in the steady state the exchange rate is equal to its fundamental. However, given that it takes a relatively long period for the exchange rate to return to its fundamental value, in a stochastic environment, the exchange rate will often be attracted by temporary equilibria that deviate from the fundamental. This will then lead to relatively long episodes of disconnection. These phenomena become more pronounced when the size of the stochastic shocks increases and when the chartists' extrapolation parameter $\beta$ increases. It is also for these values that we obtain the bubble and crash scenarios illustrated in the previous section. 


\subsection{The role of the discount factor in both models}

Since the disconnection under statistical learning occurs in the high frequency case, it appears that the key parameter in the model is the discount factor $\alpha$. Therefore, we analyze the sensitivity of the results of the different models with respect to the discount factor.

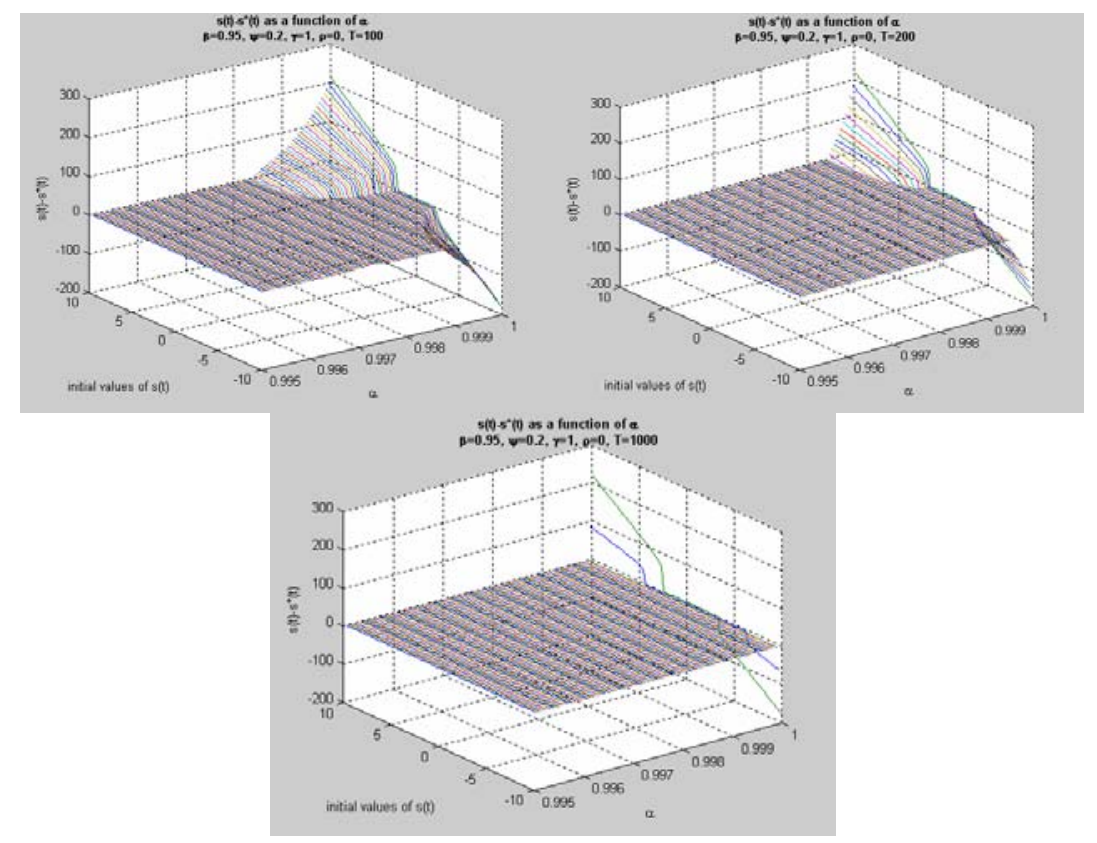

Figure 7: Difference between market and fundamental rates as a function of discount factor under fitness learning

As before, we simulated the model assuming that the stochastic realizations of the fundamental was identical for all the different values of the discount factor $\alpha$ and for all initial conditions. We varied the values of the discount factor $\alpha$ between 0.995 and 1 . We first show the results for the fitness learning model in Figure 7. As in the previous section we show results for different simulation periods, i.e. $T=100, T=200$ and $T=1000$. On the vertical axis we set out the deviation of the exchange rate from its fundamental. On the x-axis we show the different values of the discount factor $\alpha$, and on the y-axis the different initial conditions. We find first that when the model is simulated over short periods of time $(T=100)$, the exchange rate can deviate significantly from 
its fundamental. Thus, it appears that when the initial shock is large enough and when $\alpha$ is large enough (high frequency), the exchange rate remains disconnected from its fundamental value. We observe that the exchange rate can get caught in a (temporary) non-fundamental equilibrium. When we allow the simulation period to increase we observe that the "zone of disconnection" shrinks. When $T$ is large enough only values of $\alpha$ close enough to 1 will produce an exchange rate that is disconnected from the fundamental.

We performed a similar sensitivity analysis for the statistical learning model. The results are presented in Figure 8. We find that when the simulation period is short $(T=100)$ the exchange rate deviates from its fundamental and this deviation increases with $\alpha$. As the simulation period is increased one needs larger $\alpha^{\prime} s$ for the exchange rate to deviate from its fundamental. When $T=1000$ the exchange rate converges to its fundamental for all but the values of $\alpha$ very close to 1 . Thus, as agents have more observations to learn the true parameters, the exchange rate will be pulled to its fundamental.

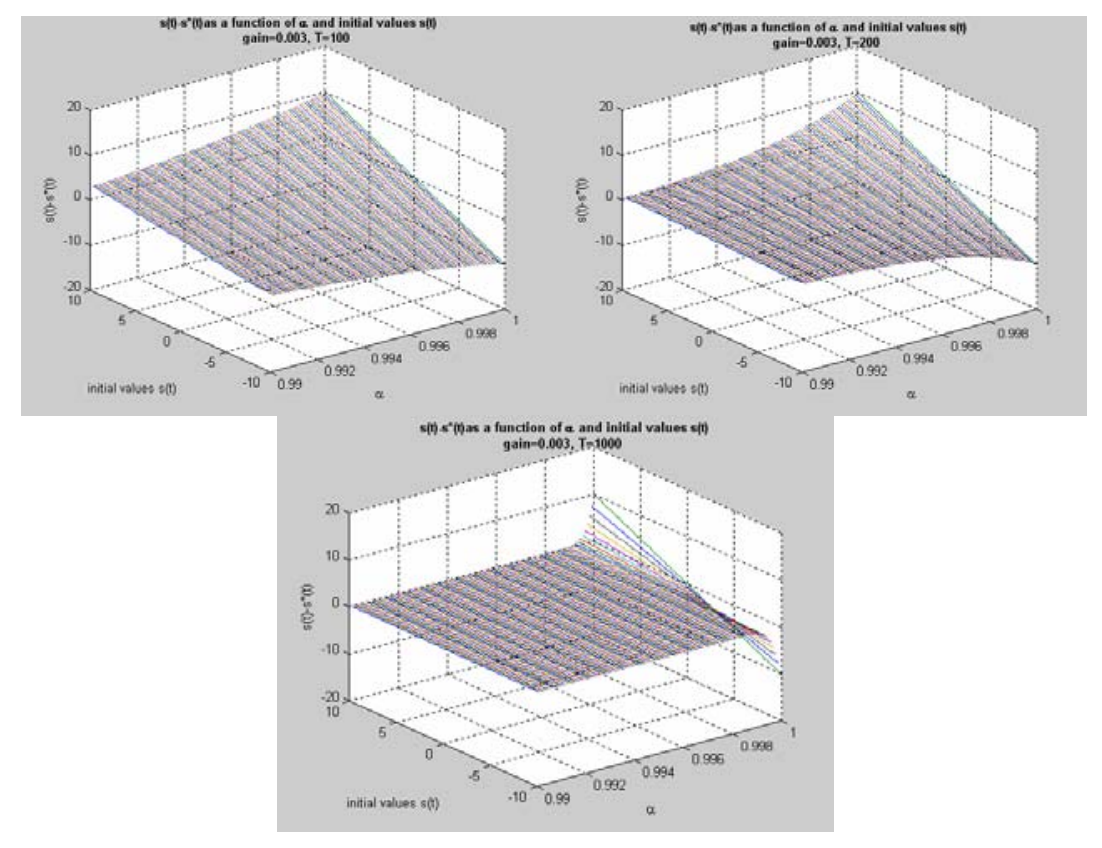

Figure 8: Difference between market and fundamental rates as a function of discount factor under statistical learning 
Thus, the results obtained for the fitness and statistical learning models are similar. There are, some differences though. First, the deviations from the fundamental take much larger proportions in the fitness learning than in the statistical learning model. Second, in the fitness learning model there is a clear discontinuity that separates the space of fundamental equilibria from the space of (temporary) nonfundamental equilibria. This explains why in the fitness model a relatively small shock in the initial condition can bring the exchange rate into a non-fundamental (bubble) equilibrium. In the statistical learning model, the transition between fundamental and non-fundamental equilibria is smooth, so that one needs a relatively large shock in initial conditions to drive the exchange rate away from its fundamental.

\section{Descriptive statistics}

In this section, we analyze the variability characteristics of the market exchange rate produced by the model under two different learning mechanisms. For this purpose, we use monthly market rates of the German Mark, Japanese Yen and UK Pound, during the period between the sixth month of 1982 and the twelfth month of 1998. These market rates are expressed as units of these currencies per US dollar and they are provided by the IMF in the International Financial Statistics. The monthly fundamental rates are constructed on the basis of the monetary model:

$$
s_{t}^{*}=\left(m_{t}-m_{t}^{*}\right)-\phi\left(y_{t}-y_{t}^{*}\right)
$$

where $s_{t}^{*}$ denotes the log fundamental rate expressed as units of national currency per US dollar, $m_{t}, m_{t}^{*}$ and $y_{t}, y_{t}^{*}$ are money supplies and real incomes in the home and foreign countries, respectively. The income elasticity of money demand $\phi$ is assumed to be 1 . The money supply is proxied by seasonally adjusted M2 aggregates coming from OECD Main Economic Indicators Database. For the real income data, we use seasonally adjusted industrial production from the same database. We set $\alpha=0.996$ $\left(\alpha=\frac{1}{(1+0.05)^{\frac{1}{12}}}=0.996\right)$ which corresponds to monthly frequencies and $\gamma=0.003$ (which as before assumes that agents use data extending over 14 years). Table 1 shows the unconditional volatility of the market and fundamental rates for three currencies and of the simulated series of the market exchange rate under fitness and statistical learning. 
Table 1: Volatility in the exchange rate market

\begin{tabular}{|c|c|c|c|}
\hline & Market volatility & Fundamental volatility & Excess Volatility \\
\hline DM & 0.027 & 0.015 & 0.012 \\
JY & 0.030 & 0.014 & 0.016 \\
UKP & 0.027 & 0.013 & 0.014 \\
\hline Statistical & & & \\
Learning & 0.015 & 0.015 & 0 \\
\hline Fitness & & & \\
Learning & 0.021 & 0.015 & 0.006 \\
\hline
\end{tabular}

Return volatility is measured by a sample standard deviation of the returns. DM denotes the German Mark, JY the Japanese Yen and UKP the UK Pound. The excess volatility is calculated as a difference between the market return volatility and the fundamental return volatility.

The unconditional volatility is measured as a sample standard deviation of the returns. In the simulations we set the standard deviation of the fundamental rate equal to 0.015 (remember that the fundamental rate follows a random walk eq.(3)), which is a number corresponding to the standard deviation found in the data (see the first three columns of Table 1). We notice that the statistical learning model does not generate excess volatility. In contrast, the fitness learning mechanism produces exchange rate returns more volatile than the fundamental returns.

\subsection{Excess volatility with fast statistical learning}

The previous results of the statistical learning model assumed $\gamma=0.003$. This value implies a relatively slow learning process in that agents collect a long series of data points. We experimented with higher value of $\gamma=0.015$ (implying a faster learning) still assuming high frequency i.e. $\alpha=0.996$. We found two possible cases. First, as already observed in Section 5, we find that the market exchange rate can be disconnected from the fundamental rate when the parameters $\psi$ and $\beta$ do not converge to the steady state values. Furthermore, a higher gain produces an excess volatility of 0.01 which is a number close to the one observed in reality. The excess volatility generated in an environment where the agents update the parameters of the model using statistical methods has also been found by Kim and Mark (2005). Second, a higher gain can also lead to instability of the model. 


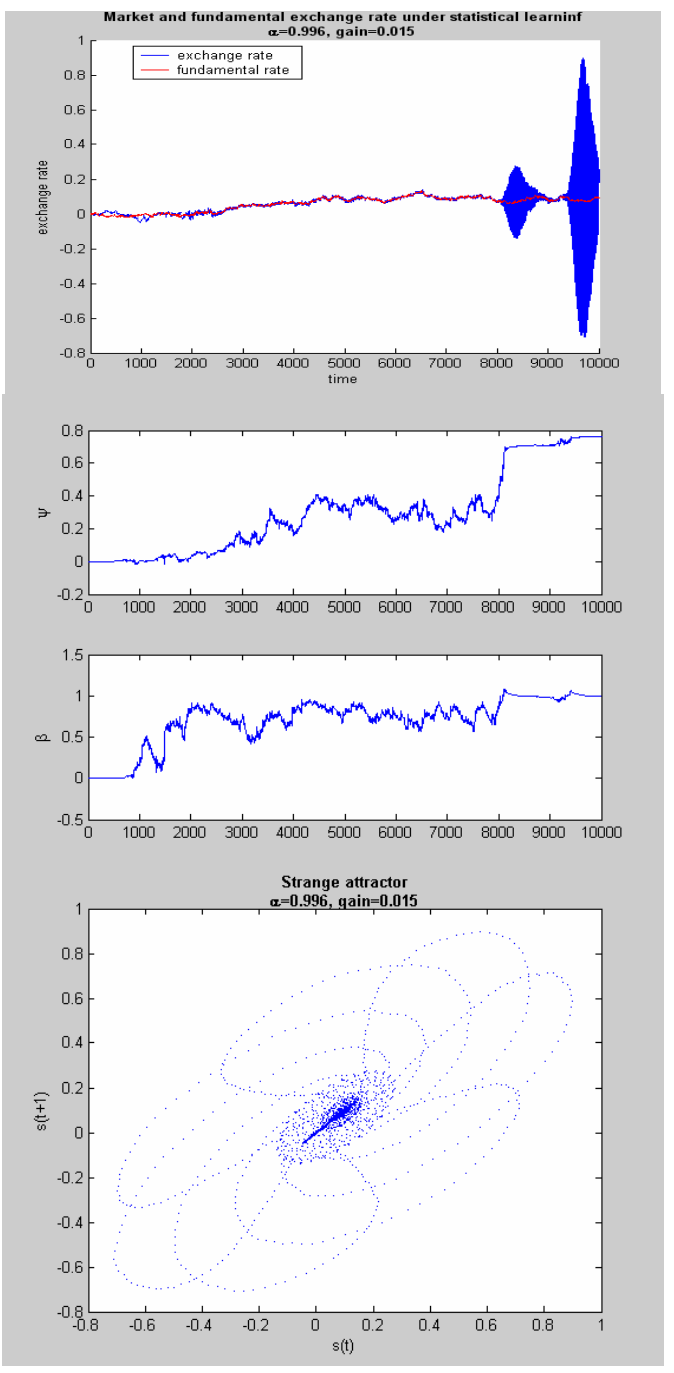

Figure 9: Explosive solution under statistical learning 
We illustrate this in Figure 9. The upper part of the figure shows the exchange rate in the time domain. It can be seen that the exchange rate is sometimes gripped by episodes of large turbulence. The underlying reason is that agents learn too high a value of the extrapolative parameter $\beta$ (which tends to converge to 1 ) and too low a value of the mean-revering parameter $\psi$ (see the second and third panels of Figure 9 ). Thus, the combination of high frequency observations and relatively fast learning can create high turbulence. This is also a case when the volatility of the market exchange rate is extremely high. The excess volatility of the exchange rate showed in Figure 9 is equal to 5.9 , a number that largely exceeds the empirically observed excess volatility (see Table 1).

The lower panel in Figure 9 shows the phase diagram corresponding to the simulations of the exchange rate (top panel). It describes the movements of the exchange rate (the attractor) in the $s_{t}, s_{t-1}-$ space. We obtain a very complex attactor which is responsible for the high volatility.

When we allow $\gamma$ to increase beyond 0.02 the models leads to explosive solutions. Thus, a combination of high discount factor $\alpha$ and high constant gain $\gamma$ leads to instability. We conclude that fast statistical learning can generate excess volatility close to the one observed in the data. However, it can also produce volatility that largely exceeds the empirically observed volatility. It can even lead to explosive solutions.

\section{Conclusion}

In this paper, we investigated the behavior of the exchange rate within the framework of a standard asset pricing model. We introduced into this model boundedly rational agents who use simple rules to forecast the future exchange rate. We assumed that agents test these rules continuously. This testing procedure is the mechanism by which discipline is imposed on the behavior of individual agents. We specified two alternative testing procedures (learning mechanisms). In the first one, agents select the rules based on a fitness method. This mechanism assumes that agents evaluate the two forecasting rules by computing their past profitability and to increase (reduce) the weight of one rule if it is more (less) profitable than the alternative rule. In the second mechanism, agents learn to improve these rules using statistical methods. They are assumed to have 
some basic knowledge of econometrics, such that they estimate the parameters of the rules they use.

We investigated which of these learning rules generates more realistic dynamics of the exchange rate. In particular, we focused on two regularities observed in the foreign exchange markets, namely the disconnect puzzle and the excess volatility phenomenon. For this purpose, we carried out a number of analytical and numerical exercises. Our results can be summarized as follows.

First, we found that both learning mechanisms are efficient in revealing the fundamental value of the exchange rate in the steady state.

Second, both mechanisms can generate the disconnection of the market rate from the fundamental value and the excess volatility of the market returns with respect to fundamental returns. The fitness learning model produces the disconnect phenomenon of the exchange rate when it is applied to low and high frequencies of the observations. The disconnection produced by the model are large and persistent, indicating the presence of bubbles and crashes. As the sensitivity analysis demonstrated, these disconnections are mainly triggered by extrapolative forces of the chartist forecasting rule.

The statistical learning model does not reproduce the disconnection phenomenon when it is applied to low frequency observations. The model does generate disconnections when applied in an environment of high frequency observations. This disconnection arises because, at high frequencies, the signal to noise ratio is very low. A lack of data makes the agents unable to find the steady state values of the model parameters. When we simulate the model over sufficiently long periods, agents accumulate more data and are capable of learning the true parameter values of the underlying model. The disconnection then tends to disappear. Thus under learning the disconnect phenomenon should be considered as a temporary phenomenon.

Finally, we analyzed in detail the variability characteristics of the market exchange rate produced by the model under the two different learning mechanisms, and we compared these with those observed in the data. Fitness learning generates excess volatility which however remains smaller than the one observed in the data. Statistical learning, however, only generates excess volatility with a narrow set of values for the discount factor and the constant gain. 
More research needs to be done to test the validity of these two learning methods. In particular we intend to analyze other statistical properties of the exchange rate movements generated by the two models (e.g. volatility clustering and excess kurtosis) and to confront these to the observed movements of the exchange rate. This will allow us to evaluate the two modelling approaches with more confidence. 


\section{References}

[1] Anderson, S., de Palma, A., Thisse, J.-F., (1992), Discrete Choice Theory of Product Differentiation. MIT Press, Cambridge, Mass.

[2] Bénassy-Quéré A., Larribeau S., Macdonald R. (1999), Models of Exchange Rate Expectations: Heterogeneous Evidence from Panel Data. CEPII Working Paper $99-03$.

[3] Brock, W., and Hommes, C., (1997), A Rational Route to Randomness. Econometrica, 65, 1059-1095

[4] Brock, W., and Hommes, C.,(1998), Heterogeneous beliefs and routes to chaos in a simple asset pricing model. Journal of Economic Dynamics and Control, 22, $1235-1274$.

[5] Cheung, Y. et al., (2004), How Do UK-Based Foreign Exchange Dealers Think Their Market Operates? International Journal of Finance \& Economics, 9(4).

[6] Cheung, Y. and Chinn M., (2001), Currency Traders and Exchange Rate Dynamics: A Survey of the U.S. Market, Journal of International Money and Finance, 20(4), 439-471.

[7] Cheung Y. and Friedman D., (1997), Individual Learning in Games: Some Laboratory Results, Games and Economic Behavior, 19(1), 46-76.

[8] Chinn M. and Meese R., (1995), Banking on currency forecasts: How predictable is change in money? Journal of International Economics 38, 161-178.

[9] De Grauwe, P. and Grimaldi M., (2005), The Exchange Rate and its Fundamentals in a Complex World. Review of International Economics, 13 (3), 549-575.

[10] De Grauwe, P., and Grimaldi, M. (2006), The exchange rate in a behavioral finance framework. Princeton University Press, (forthcoming). 
[11] Evans, G., and Honkapohja, S., (2001), Learning and Expectations in Macroeconomics. Princeton University Press, 421pp.

[12] Frankel J., and Froot K., (1987),

[13] Frankel J., and Froot K., (1990), Chartists, Fundamentalists, and Trading in the Foreign Exchange Market, American Economic Review, 80, (2), 181-185.

[14] Fudenberg D. and Levin D., (1998), Theory of Learning in Games. MIT Press.

[15] R. W. Hafer; Scott E. Hein, (1984), Financial Innovations and the Interest Elasticity of Money Demand: Some Historical Evidence: Note, Journal of Money, Credit and Banking, 16 (2), 247-252.

[16] Kahneman, D., and Tversky, A., (1973), Prospect Theory: An analysis of decisions under risk. Econometrica, 47, 313-327.

[17] Kahneman, D., (2002), Maps of Bounded Rationality: A Perspective on Intuitive Judgment and Choice. Nobel Prize Lecture, December 8, Stockholm.

[18] Kim Y., Mark N., (2005), Exchange Rates and Fundamentals under Adaptive Learning. mimeo.

[19] Kirman, A., (1993), Ants, rationality and recruitment. Quarterly Journal of Economics, 108: 137-156.

[20] Lux, T., and Marchesi, M., (1999), Scaling and Criticality in a Stochastic MultiAgent Model of a Financial Market. Nature, 397, 498-500.

[21] Mark, Nelson C. , (1995), Exchange Rates and Fundamentals: Evidence on LongHorizon Predictability. The American Economic Review, 85 (1), 201-218.

[22] Milani F., (2005), Expectations, Learning and Macroeconomic Persistence. Princeton University, mimeo.

[23] Sarno, L., and Taylor, M., (2002), The economics of exchange rates, Cambridge University Press, 318 pp. 
[24] Taylor M. and Allen H., (1992), The Use of Technical Analysis in the Foreign Exchange Market, Journal of International Money and Finance, 11, pp. 304-314. 


\section{Appendix A}

Table 2: Parameters values in time domain simulations

\begin{tabular}{|c|c|c|c|c|c|}
\hline & \multicolumn{5}{|c|}{ Simulations/Figures } \\
\hline Parameters & Fig 1 & Fig 2 & Fig 3 & Fig 4 & Fig 5 \\
\hline & & & & & \\
$\alpha$ & 0.988 & 0.988 & 0.999 & 0.999 & 0.999 \\
$\psi$ & 0.25 & & 0.004 & & \\
$\beta$ & 0.95 & & 0.95 & & \\
$\sigma_{\eta}$ & 0.03 & 0.03 & 0.0033 & 0.0033 & 0.0033 \\
$\sigma_{\epsilon}$ & 0.03 & 0.03 & 0.0033 & 0.0033 & 0.0033 \\
$\delta$ & 10 & & 10 & & \\
$\mu$ & 1 & & 1 & & \\
$\gamma$ & & 0.02 & & 0.0003 & 0.0003 \\
$T$ & 1000 & 1000 & 1000 & 1000 & 1000 \\
\hline
\end{tabular}


Table 3: Parameters values in sensitivity analysis

\begin{tabular}{|c|c|c|c|c|c|c|}
\hline & \multicolumn{5}{|c|}{ Simulations/Figures/Tables } \\
\hline Parameters & Fig 6 & Fig 7 & Fig 8 & \multicolumn{2}{|c|}{ Table 1} & \multicolumn{2}{|c|}{ Fig 9} \\
\hline & & & & Stat & Fit & \\
$\psi$ & 0.999 & $0.995-1$ & $0.99-1$ & 0.996 & 0.996 & 0.996 \\
$\beta$ & 0.2 & 0.2 & & & 0.01 & \\
$\sigma_{\eta}$ & $0.5-1$ & 0.95 & & & 0.95 & \\
$\sigma_{\epsilon}$ & 0.1 & 0.1 & 0.1 & 0.015 & 0.015 & 0.015 \\
$\delta$ & 0.1 & 0.1 & 0.1 & 0.015 & 0.015 & 0.015 \\
$\mu$ & 5 & 5 & & & 10 & \\
$\gamma$ & 1 & 1 & & & 1 & \\
$\psi_{0}$ & & & 0.003 & 0.003 & & 0.015 \\
$\beta_{0}$ & & & 0 & 0 & & 0 \\
$s_{0}$ & $(-10)-(+10)$ & $(-10)-(+10)$ & $(-10)-(+10)$ & 0 & 0 & 0 \\
$\omega^{f}$ & 0.5 & 0.5 & & & 0.5 & \\
$\omega^{c}$ & 0.5 & 0.5 & & & 0.5 & \\
$T$ & $50-1000$ & $100-1000$ & 100 & 200 & 200 & 1000 \\
\hline
\end{tabular}

Stat corresponds to statistical learning procedure and Fit to the fitness learning method. 